\title{
Work in Progress:Development of Hands-on Desktop Learning Modules for Bioengineering Courses
}

\section{Mr. Amirhossein Arasteh, Washington State University}

Mr. Amirhossein Arasteh obtained his bachelor's degree from Sharif University of Technology, Tehran, Iran, in 2012 and has some experience working in automotive lubricant oil industry. He is currently working on a Ph.D. that includes the selective separation of prostate circulating tumors cells.

\section{Casey M. Clark, Washington State University}

Casey Clark recently completed her undergraduate studies at Montana Tech of the University of Montana where she obtained three bachelor of science degrees in Environmental Engineering, Mathematical Sciences, and Statistics. In the fall of 2012, Casey worked under the direction of Dr. Bernard Van Wie at Washington State University to develop bioengineering Desktop Learning Modules and to research the separation of prostate circulating tumor cells within the blood, pursuant to Ph.D. studies in Engineering Science. She is currently an Adjunct Professor at Montana Tech in the Mathematical Science department.

\section{Mr. Bernard J. Van Wie, Washington State University}

Prof. Bernard J. Van Wie did his B.S., M.S. and Ph.D. and postdoctoral work at the University of Oklahoma where he also taught as a visiting lecturer. He has been on the Washington State University faculty for 30 years. For the past sixteen years he has focused strongly on innovative pedagogy and done technical research in biotechnology. His recent Fulbright Exchange to Nigeria set the stage for his receipt of the Marian Smith Award, an award given annually to the most innovative teacher at WSU.

\section{Dr. Nehal I. Abu-lail, Washington State University \\ Dr. Olusola Adesope, Washington State University-Pullman}

Dr. Olusola O. Adesope is an assistant professor of Educational Psychology at Washington State University, Pullman. His research is at the intersection of educational psychology, learning sciences, and instructional design and technology. His recent research focuses on the cognitive and pedagogical underpinnings of learning with computer-based multimedia resources; knowledge representation through interactive concept maps; meta-analysis of empirical research, and investigation of instructional principles and assessments for engineering designs. Dr. Adesope holds a Ph.D. in Educational Psychology and a M.Sc. in Educational Technology from Simon Fraser University, Canada.

\section{Dr. Shane A. Brown P.E., Washington State University}

Dr. Shane Brown conducts research on cognition and conceptual change in engineering. He received his bachelor's and Ph.D. degrees from Oregon State University, both in Civil Engineering. His Ph.D. degree includes a minor in science and mathematics education. His master's degree from the University of California, Davis is in Environmental Engineering. Dr. Brown is a licensed professional civil engineer and has six years of experience designing water and waste water treatment facilities in central California. He was the recipient of the NSF CAREER award in 2011. Dr. Brown's research focuses on theoretical approaches to understanding why some engineering concepts are harder to learn than others, and how the concepts are embedded in contexts. 


\title{
A Work in progress- Development of Hands-on Learning Modules for
}

\author{
Bioengineering courses
}

\begin{abstract}
Current engineering courses heavily rely on traditional lectures to cover engineering concepts. The engineering education community, however, recognizes that this is not the best method for teaching engineering materials. Our team has focused on expanding the use of well researched, though still new to engineering, teaching pedagogies of hands-on, active and problem-based learning through the development of Desktop Learning Modules (DLMs).

The DLMs contain miniaturized processes such as shell and tube or double pipe heat exchangers (chemical engineering) and hydraulic flow channels (civil engineering). A unique expandable electronic system within the DLM connects with a sensor system, allowing cartridges to be interchanged. We are expanding applications by creating new cartridges for teaching bioengineering concepts. One such cartridge will simulate cell sedimentation, particularly applied to the separation of prostate circulating tumor cells (PCTCs) within human blood. This cartridge mimics the partial separation of cells in an elutriator or apheresis device. For this a cylindrically shaped elutriator containing millimeter-sized polymer particles of variable size and density is being used to mimic hindered settling and help students understand concepts related to separation of PCTCs from white blood cells.
\end{abstract}

The cell separation DLM is being implemented into a Fluid Mechanics \& Heat Transfer course ChE 332 in the spring 2013 semester. Assessment consists of the use of concept tests before and after use of the DLM to determine improvement in understanding basic concepts and persistence and/or repair of misconceptions.

A physical model of the cell separations DLM and preliminary classroom assessment data will be presented at the 2013 ASEE Meeting as a "work in progress". Future work will include development and implementation of cell sensor and thermoregulation cartridges.

\section{Introduction}

Our main goal is to simulate cell separation in a DLM, so that students may engage in a hands-on interactive, problem based learning experience. We are developing assessments to be given before and after running the DLMs to determine improvements in basic concepts pertaining to cell separations.

\section{DLM Sedimentation Cartridge}

As shown in Fig. 1 the cell separation cartridge consists of a tube capped with metal meshing at both ends, with a selection of plastic beads of different sizes and densities placed in the tube to mimic different cells. A small water tank is attached to the 
apparatus. Water is pumped from the tank to the tube at desired flow rates and fluidizes the beads in the tube. Small tubes are connected to taps at the two ends of the larger sedimentation tube and connected to a differential pressure transducer. The flow rate through the tube is adjustable and can be adjusted and read through use of a rotometer. The device is portable, works with rechargeable batteries, and can easily be brought into and used in the classroom.

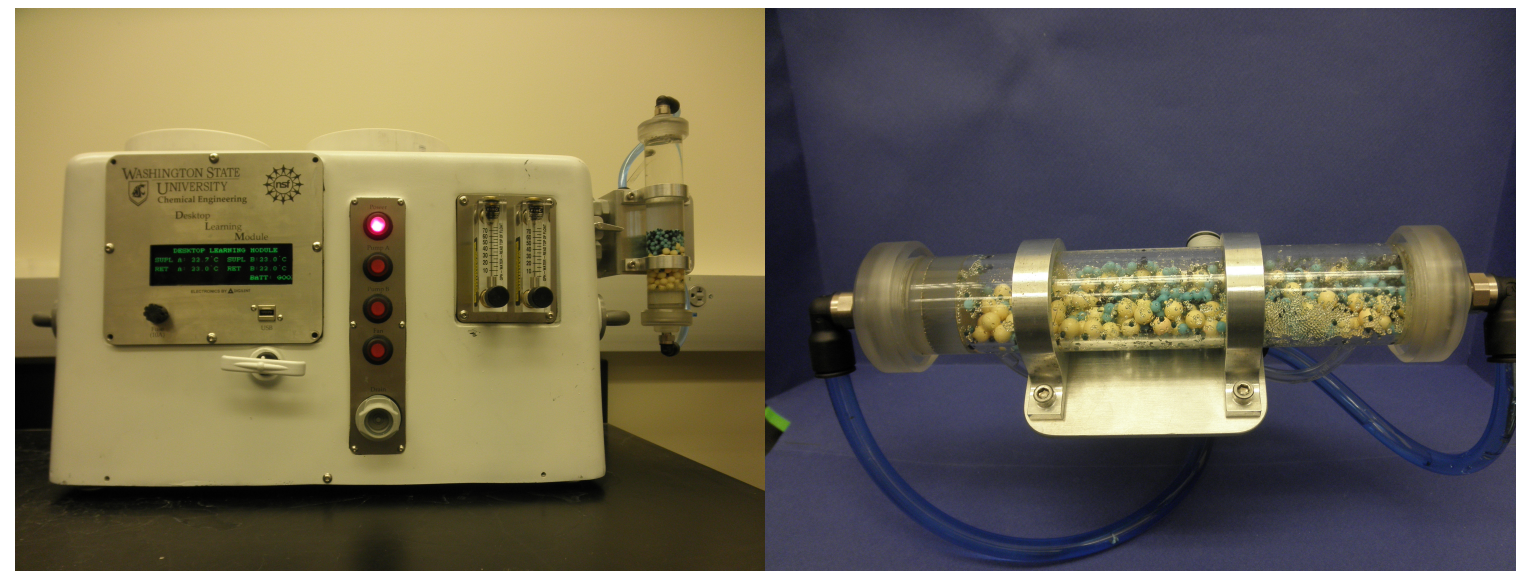

Figure 1. Desktop learning modules and the cell separation cartridge.

\section{Educational Plan}

We are preparing worksheets to help students learn basic concepts pertaining to cell separations. We used the work sheet along with the separation DLM in a Fluid Mechanics \& Heat Transfer course taught in the spring 2013 semester at Washington State University. Students are given pre- take-home and a post-quiz on principles to be learned with the DLM separations cartridge. After getting familiar with the basic concepts of cell separations and having seen the separation pattern of beads inside the tube the students were provided with preliminary study data on separation of prostate circulating tumor cells (PCTCs) from peripheral blood from a simulated prostate cancer patient blood sample.

The cell separation DLM could be alternatively used to study packed and fluidized beds. We believe that by using the separation DLM students can see and understand the following concepts.

1) The system can first be used to study packed beds by monitoring pressure.

2) Fluidization starts at a certain flow rate and total pressure drop does not change across the suspended particle bed when using higher flow rates.

3) Both cell density and size affects settling rates with size being the predominant factor.

4) The average suspension density will affect the buoyant forces.

5) Due to relative sizes of particle species one must consider an effective porosity for different cell types. This will lead to larger relative settling velocities for larger cells in dilute particle suspensions and surprisingly to larger relative settling velocities for smaller cells in dense particle suspensions. 
6) At certain dilutions an "azeotrope"-like condition occurs where both larger and smaller diameter cell species will have the same settling velocity.

\section{System Analysis}

To develop a learning module for students it is important to understand the fundamental concepts surrounding cell separations. Our plan is to take these concepts and develop both in class worksheets and pre- and post-assessments.

Basically, there are three forces acting on particles inside a separation chamber; these are the gravitational force, the buoyant force and the drag force. As most cells separation process are in the Stokes regime, we will analyze the Stokes regime where $\mathrm{Re}_{\mathrm{p}}$ is less than 1. The gravitational force is modeled as the mass times the gravitational acceleration computed as $-\frac{1}{6} \pi d_{i}^{3} \rho_{i} g$, where $d_{i}$ and $\rho_{i}$ are the particle diameter and density, respectively. The buoyant force is the resisting gravitational force of the displaced fluid computed as $\frac{1}{6} \pi d_{i}^{3} \rho_{f} g$ where $d_{i}$ is the particle diameter and $\rho_{f}$ is the density of the fluid medium which for the DLM cartridge will be water or a dilute solution of carboxymethyl cellulose $(\mathrm{CMC})$ to adjust the viscosity. The drag force can be computed as $3 \pi \mu V_{i} d_{i}$ where $d_{i}$ is the diameter of the particle and $V_{i}$ is the sedimentation velocity of the particle. This force balance leads to Stokes equation for sedimentation $V_{i}=\frac{g d_{i}^{2}\left(\rho_{i}-\rho_{f}\right)}{18 \mu}$. Stokes law for calculating the drag will require the particles be present at infinite dilution; otherwise corrections need to be made. For dense suspensions we can calculate the sedimentation settling velocity with the Masliyah ${ }^{1}$ relation for the viscous drag correction due to interparticle collisions with $V_{i}=S_{i} g\left(\frac{\rho_{i}-\rho_{\text {susp }}}{\rho_{i}-\rho_{f}}\right) \epsilon_{i}^{2.7}$ where $S_{i}$ is the sedimentation coefficient for the particle, $S_{i}=\frac{d_{i}^{2}\left(\rho_{i}-\rho_{f}\right)}{18 \mu}$ and $\epsilon_{i}$ is the apparent porosity of the particle. In order to calculate the apparent porosity for each species we use the method proposed by Patwardahan and Tien ${ }^{2}$, first applied to blood cells by Van Wie and Hustvedt ${ }^{3}$ then by Leatzow et al. ${ }^{4}$ to calculate the average weighted particle diameter $d_{\text {avg }}$ and the porosity $\epsilon$ of the fluidized bed, assuming that the highest suspended particles indicate the end of the fluidized bed. Now we are able to calculate the average void envelope thickness $d_{\epsilon}=d_{a v g}\left((1-\epsilon)^{-\frac{1}{3}}-1\right)$ and then we can assign an apparent porosity for each particle $\epsilon_{i}=1.0-\left(1.0+\frac{d_{\epsilon}}{d_{i}}\right)^{-3}$.

\section{Beads}

In our DLM we are using 4 types of beads to mimic the separation of 4 different cell types. The details of the particles used in the tube are as follows, 250 turquois beads with a diameter of $0.38 \mathrm{~cm}$ and an average density of $1.19 \frac{\mathrm{g}}{\mathrm{cm}^{3}}, 7$ beige beads with a diameter of $0.7 \mathrm{~cm}$ and an average density of $1.29 \frac{\mathrm{g}}{\mathrm{cm}^{3}}, 1$ pink bead with a diameter $1.4 \mathrm{~cm}$ and a density of $1.31 \frac{\mathrm{g}}{\mathrm{cm}^{3}}$, and 300 green beads with a diameter $0.19 \mathrm{~cm}$ and an average density 
of $1.28 \frac{\mathrm{g}}{\mathrm{cm}^{3}}$. Preliminary cartridge tests are being done with a total fluidized bed volume of the $\sim 16 \mathrm{~cm}^{3}$ and a total volume of beads of $\sim 11 \mathrm{~cm}^{3}$. The flow medium is water with a viscosity of $0.01 \frac{\mathrm{g}}{\mathrm{cm} . \mathrm{s}}$. Furthermore we use the same beige beads with a set of tiny glass beads with a diameter $0.088 \mathrm{~cm}$ and a density of $3.140 \frac{\mathrm{g}}{\mathrm{cm}^{3}}$, and some black beads with somewhat similar density and diameter to that of the turquois beads in a highly viscous solution of CMC. The above assembly has the advantage of visually showing the transition between three different regimes in separation.

\section{Results}

First we will discuss what students can learn from bead separations in an elutriation column, then we will turn our attention to PCTC separations in human blood for biomedical applications.

\section{Bead Separations}

Fig. 2 shows the predicted settling velocity results, assuming Stokes regime sedimentation, for various beads as a function of the volume percentage of beads contained in a suspension. We note three regions of interest that are used in assisting students in learning concepts: 1) the more dilute regime of $0-25 \%$ beads; 2) one of intermediate bead suspension density of $30-40 \%$; and 3) one of higher suspension density of $45 \%$ beads and above.

Within the dilute regime the particle diameter is the dominant parameter in predicting which particles separate fastest as this affects the volume upon which gravity acts, while the particle circumference affects the perimeter over which drag acts. Particles with large volume to circumference ratios $\frac{d_{i}}{6}$ will settle and separate toward the bottom of a fluidized suspension. This is demonstrated in Fig. 2 for beads volume fractions of less than $25 \%$, where particles settle according to their diameter. Students should realize that if suspension density is low, the best way to separate particles is based on respective sizes with smaller ones being eluted first from the top of a suspension, followed by the next largest, etc. They also need to realize that the buoyant term $\frac{1}{6} \pi d_{i}^{3} \rho_{f} g$ has more of an impact as the suspension density increases as one must replace $\rho_{f}$ with the average density of the particle suspension; this along with the collision effects on viscous drag cause an exponential decay in the settling velocity curves. 


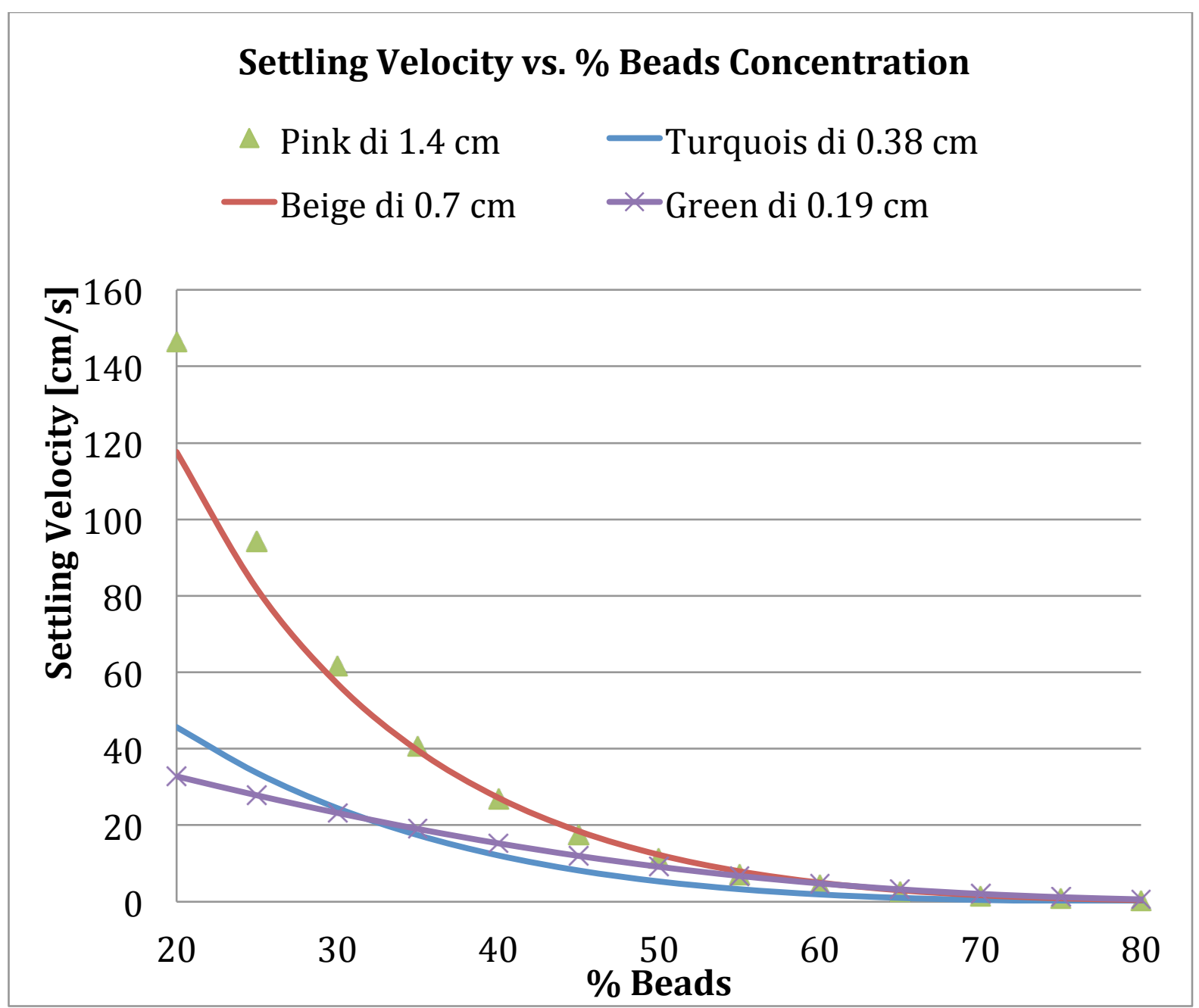

Figure 2. Predicted settling velocities for beads using Masliyah correlation.

However, it is not in every situation that one will want to separate cells at smaller suspension densities. First, particles are more dilute and therefore there are fewer numbers of separated species or one must use a larger volume apparatus to perform the separations. Secondly, many biological suspensions are more concentrated, such as blood where formed elements, i.e. cells boost suspension densities to the $35-50 \%$ range. Therefore, we wish to assist students in learning about the intermediate suspension density range. 

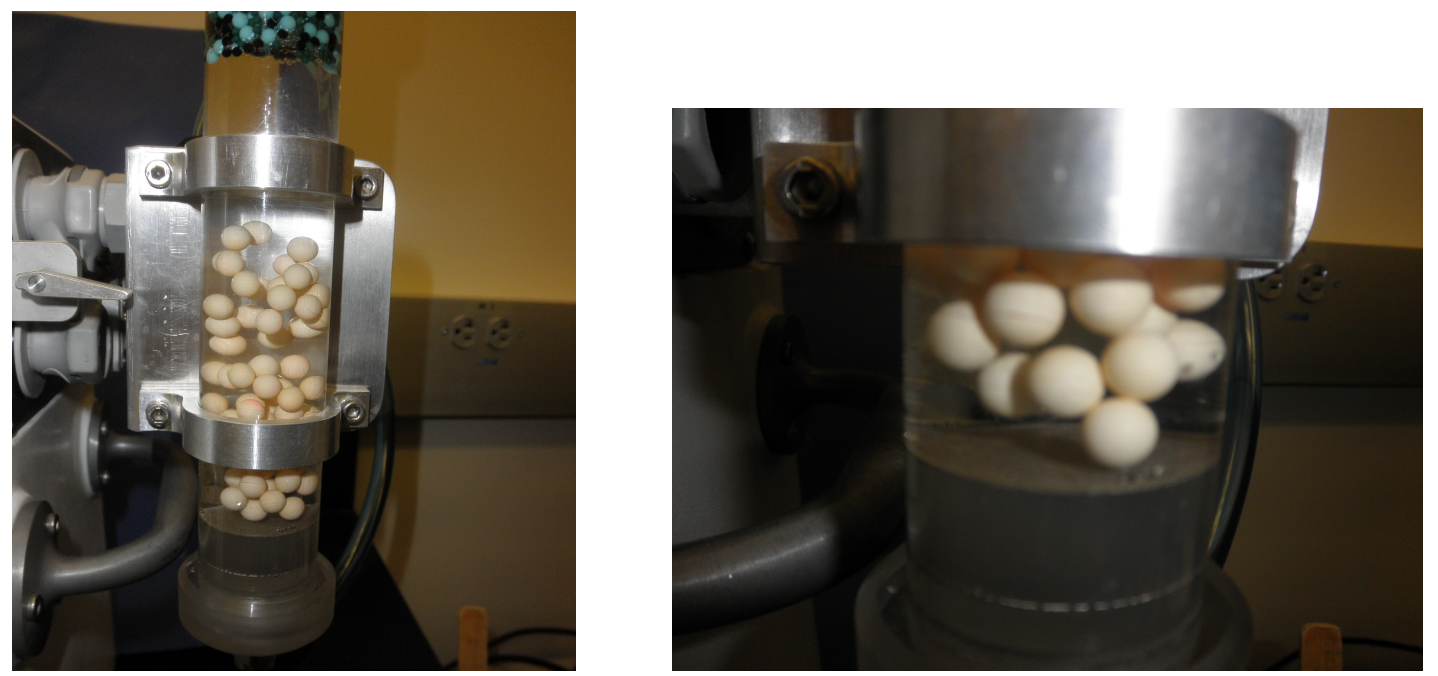

Figure 3. Separation of large beige particles towards the end of the elutriator despite the presence of more dense, but tiny glass beads.

In the intermediate bead suspension density region, $30-40 \%$ beads, the effective porosity and suspension density will cause smaller more dense particles to gain a settling advantage over those with a large volume to circumference ratio. In this region smaller particles will experience a significantly more dilute environment in comparison to larger particles. This is due to the relative size of small particles compered to interstitial volumes. Consequently smaller particles will experience a lower suspension density and they will encounter fewer interparticle collisions as well. Particle density will dominate the separation pattern, and hence alter the former separation of cells according to size. This effect can be observed in Fig. 4a where green bead settling velocity first equals, and then surpasses turquois settling velocity. Fig. $4 \mathrm{~b}$ depicts the change in settling pattern in the intermediate regime. In this region the tiny and dense glass beads will settle the fastest and towards the bottom end of the elutriator, even below the largest beige particles. Note that the black and turquoise beads represent an azeotrope condition where no separation between these two particles occurs. 

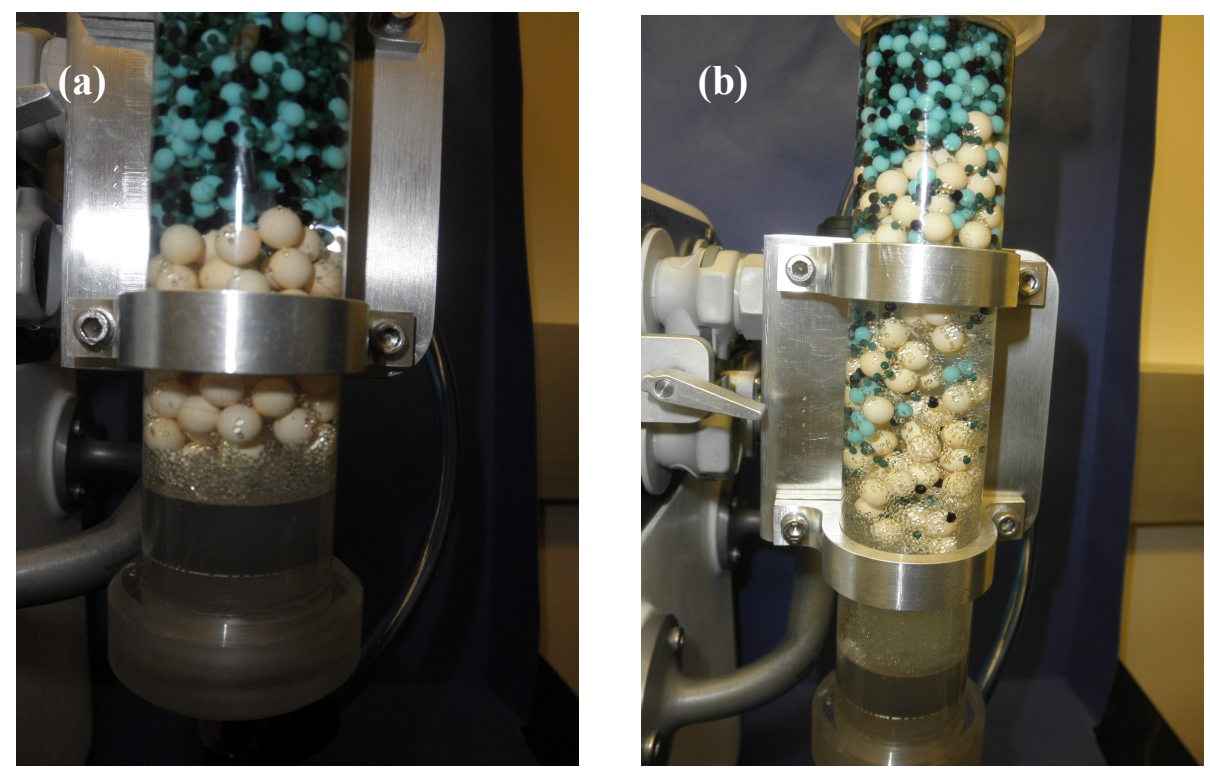

Figure 4 . Separation of the dense \& tiny glass beads towards the bottom of the chamber in the intermediate dilution regime. The figure on the left shows the intermediate dilution region at a semi-steady condition. The figure on the rights shows the fast settling of tiny glass beads at the beginning of the dilute regime note that small particles experienced fewer collisions and some have already made their way to the bottom part of the elutriator.

Analysis of the higher suspension density region of $45 \%$ beads and above shows that the settling velocities of different particles become nearly equal, and separation based on settling velocities will become difficult to achieve. This is due to the fact that at higher suspension densities the effects of the buoyant term, lesser interstitial space, and more collisions will offset each other. The resulting settling velocities are $0.47 \frac{\mathrm{cm}}{\mathrm{s}}$ for the turquois beads, $1.70 \frac{\mathrm{cm}}{\mathrm{s}}$ for the beige beads, $1.44 \frac{\mathrm{cm}}{\mathrm{s}}$ for the pink bead, and $2.05 \frac{\mathrm{cm}}{\mathrm{s}}$ for the green bead.

\section{PCTCs Separation in Human Blood}

Detection of PCTCs in the peripheral blood of a cancer patient can alert an oncologist about a malignant phenotype. We have done a preliminary study using model predictions regarding the separation of PCTCs. The same forces affecting bead separation will affect cell separations here with the exception that gravitational acceleration is replaced by centrifugal acceleration $\omega^{2} r$. For the predictions we used the Masliyah relation and took the relative ratios of blood cell types that typically exist in blood with a hematocrit of $38 \%$ and changed the amount of plasma present. We also added a small number of PCTCs, the number of them being of less importance, but typically some small fraction of the total white blood cells. Since plasma rises due to displacement by settling cells, settling velocities are determined relative to the fluid medium using the equation $V_{i, r}=V_{i}\left(1-\alpha_{i}\right)-V_{j} \alpha_{j}-V_{k} \alpha_{k}-V_{l} \alpha_{l}$ where $\alpha^{\prime} s$ are the volume fractions of particles and $V^{\prime} s$ are settling velocities. 
Fig. 5 shows the model predictions. In blood cell separation, the previous 3 regions of bead suspension density can be replaced by percent hematocrit. The first region of dilute suspension density or in this case lower HCT shows the same pattern as discussed previously, where all particles separate according to their diameter, with PCTCs having a diameter of $3.75 \mathrm{E}-3 \mathrm{~cm}$, white blood cells a diameter of $1.50 \mathrm{E}-3 \mathrm{~cm}$, red blood cells a diameter of $5.40 \mathrm{E}-4 \mathrm{~cm}$, and platelets a diameter of $2.50 \mathrm{E}-4 \mathrm{~cm}$. The second and third regions show the same behavior as with the beads with the exception that the smaller red blood cell are predicted to have settling velocities surpassing those for all other cells after about 30\% hematocrit. This behavior will be present in human blood, as red blood cells tend to coalesce and form aggregates called rouleaux. Theses rouleaux are so large that the gravitational force will dominate over drag forces.

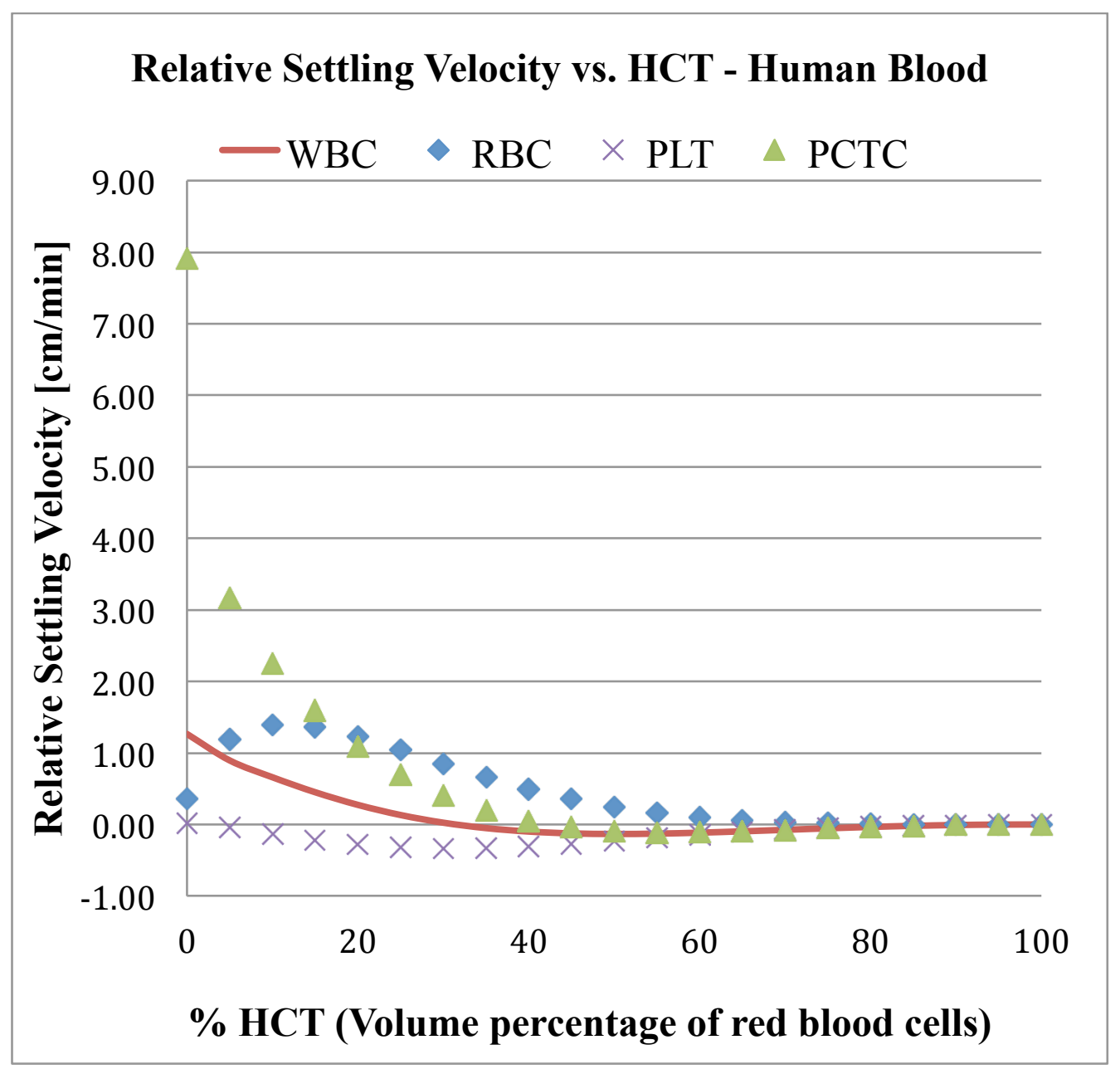

Figure 5. Predicted settling velocities of blood cells and PCTCs.

\section{Assessment}

Assessment in our fluid mechanics and heat transfer class is ongoing, where we have already given pre- and take-home-quizzes and plan to implement a post-assessment quiz 
near the semester's end. Relative gains in understanding of the sedimentation concepts will be assessed and presented at the ASEE Annual Conference.

\section{Conclusion}

The cell separation DLM device is a user friendly, rechargeable and portable learning device. It could be used to teach and show engineering students cell separation concepts. The device could be easily altered to teach fluid mechanics in a packed bed configuration or one with flow past immersed objects just by changing bead types. In this regard, we recommend the use of this device for teaching cell separation concepts.

\section{Acknowledgements}

We acknowledge support from the National Science Foundation through CCLI Grant DUE-1023121. We further acknowledge the staff and students of the Gene \& Linda Voiland School of Chemical and Bioengineering for support in helping to build, test and implement the DLMs. Finally, we acknowledge the important design contributions and manufacturing of the DLMs by Gary Held, Machinist in the WSU College of Engineering and Architecture Machine Shop.

\section{Bibliography}

1. Masliyah, J.H. Hindered settling in a multi species particle system. Chemical Engineering Science 34, 1166-1169, 1979.

2. Patwardhan, V.S. and Tien, C. Sedimentation and liquid fluidization of solid particles of different sizes and densities. Chemical Engineering Science 40, 1051-160, 1985.

3. Van Wie, B.J. and Hustvedt, E.L., Particle Interaction Effects on Blood Cell Sedimentation and Separations, Biorheology, 25, 651-662, 1988.

4. Leatzow, D.M., Van Wie, B.J., Weyrauch, B.N., and Tiffany, T.O., Design Optimization and Characterization of a Small-Scale Centrifugal Cell Separator, Analytica Chimica Acta, 435, 299-307, 2001. 